Cecília Pereira de Souza

Paulo Roberto Bastos Ri bei ro

Carlos Tito Guimarães

Liana K. Jannotti-Passos

Marilza Antunes de Souza

\title{
Esquistossomose: nova ocorrência de Biomphalaria straminea em Belo Horizonte, Minas Gerais
}

\author{
Schistosomiasis: new occurrence of Biomphalaria \\ straminea in Belo Horizonte, Minas Gerais
}

Centro de Pesquisas René Rachou, Fundação Oswaldo Cruz. Av. Augusto de Lima 1715, C. P. 1743, Belo Horizonte, MG 30290-002, Brasil.
A bstract In Belo Horizonte, Minas Gerais, Brazil, the snail Biomphalaria straminea was found in the Pampulha region. Recently the snail was found in ditches in the old Santa Lúcia Reservoir, a now-extinct focus of schistosomiasis transmission by B. glabrata. The snails were collected and examined to verify whether they were naturally infected with Schistosoma mansoni. Negative specimens were used for breeding or infection with the LE strain of S. mansoni from the laboratory and another strain obtained from eggs found in the feces of a schoolchild (VGS) from Belo Horizonte. Among the 1890 snails collected from 1994 to 1995, none were infected with S. mansoni. Among 87 snails collected and exposed to the LE strain, 9 (10.3\%) shed cercariae; among 83 snails from F1, and exposed to LE, 10 (12.0\%) shed cercariae. Among 88 snails exposed to VGS, 10 (11.3\%) shed cercariae. In Bel o Horizonte, schistosomi asi s is transmitted by B. glabrata and B. tenagophila. However, currently there exists the risk of appearance of a focus in which $B$. straminea could be the host if prophylactic measures are not taken by the authorities responsible for the construction of a park and lake at that site.

Key words Schistosomiasis; Biomphalaria straminea; Disease Vectors; Schistosoma mansoni

Resumo Em Belo Horizonte, Minas Gerais, Brasil, a Biomphalaria straminea é encontrada na região da Pampulha. Recentemente, o molusco foi encontrado em valas da antiga Barragem de Santa Lúcia, foco extinto de esquistossomose transmitida por B. glabrata. Os moluscos foram coletados eexaminados para verificar se estavam natural mente infectados com Schistosoma mansoni. Os exemplares negati vos foram usados para criação ou infecção com a cepa LE deS. mansoni, mantida no laboratório, e outra cepa VGS, obtida de ovos de fezes de escol ar de Belo Horizonte. Dentre 1.890 moluscos capturados em 1994 e 1995, nenhum estava infectado com S. mansoni. Dentre 87 exemplares coletados no criadouro e expostos à cepa LE, nove (10,3\%) eliminaram cercárias; dentre 83 mol uscos da F1, dez (12,0\%) eliminaram cercárias e dentre 88 exemplares col etados e expostos à cepa VGS, dez (11,3\%) eli mi naram cercárias. Em Belo Horizonte, a esquistossomose étransmitida por B. glabrata eB. tenagophila. Entretanto, atualmente existe o risco de aparecimento de novo foco, no qual a B. straminea poderá vir a ser a transmissora, se me didas profiláti cas adequadas não forem tomadas pelas autori dades responsáveis pel a construção de um parque elago no local.

Palavras-chave Esquistossomose; Biomphalaria straminea; Vetores de Doenças; Schistosoma mansoni 


\section{Introdução}

A Biomphalaria straminea é um vetor importante da esquistossomose no Nordeste do Brasil, onde apresenta taxas baixas de infecção e alta densidade nos criadouros (Alencar et al., 1978).

Em Minas Gerais, já foi assinalada a presença de Biomphalaria straminea em 74 municípios (Souza, 1993). Há evidência do envolvimento dessa espécie na transmissão da esquistossomose em al gumas local idades do Estado (Carvalho et al., 1987); entretanto, ainda não foram capturados exemplares com infecção natural nessa região. As taxas de infecção experi-

Figura 1

Localização dos criadouros de Biomphalaria straminea no Município de Belo Horizonte, Minas Gerais.

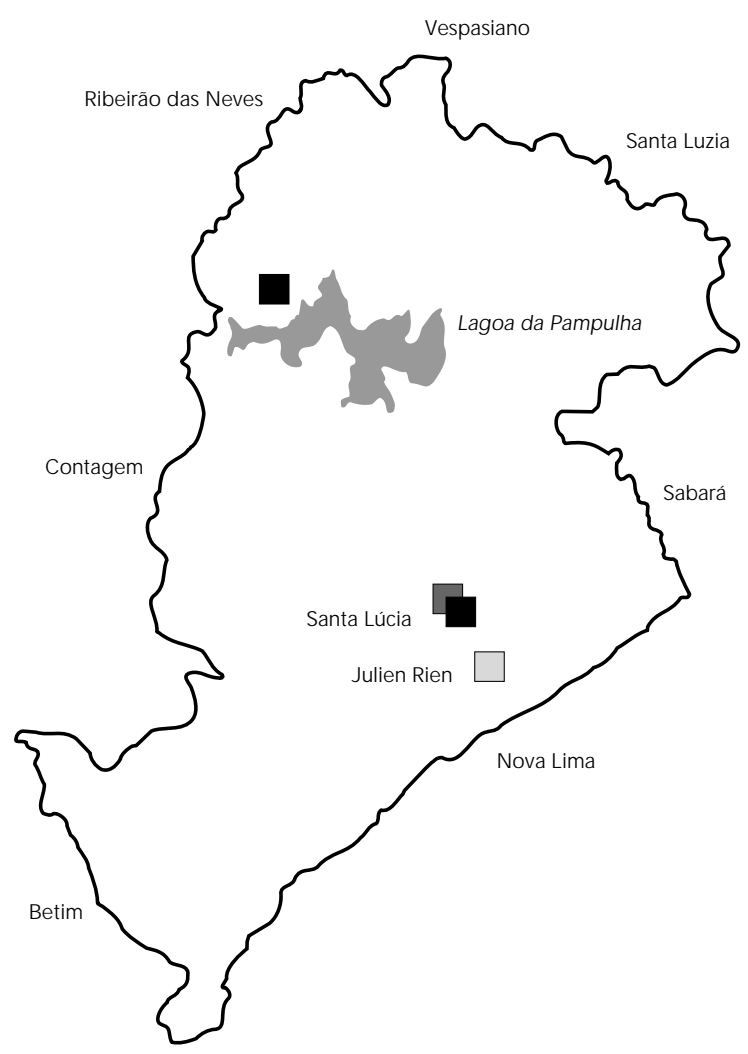

Parque implantado

Parque não implantado

Criadouro de B. straminea

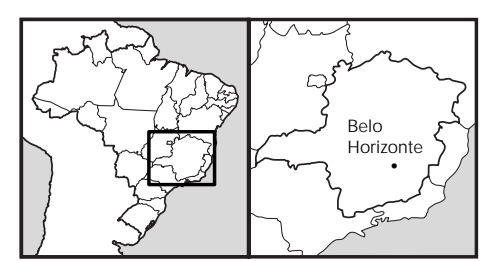

mental dos moluscos de várias localidades do Estado de M inas Gerais normalmente não ultrapassam 10\% (Freitas et al., 1972; Gerken et al., 1975; Souza et al., 1981b; 1981c; 1983).

Em Belo Horizonte, MG, a ocorrência de Tropicorbis centimetralis (=Biomphalaria straminea) foi registrada pela primeira vez por Lutz (1934). Entretanto, Martins (1938) afirmou não ter encontrado essa espécie em Belo Horizonte, resultado confirmado posteriormente por Paraense \& Santos (1950) em levantamento malacológico dessa região. Cerca de trinta anos depois destas pesquisas, em levantamento malacológico realizado por Souza et al. (1981a), foi confirmada a presença de B. straminea em Belo Horizonte. O molusco foi encontrado em sítio próximo do lago da Pampulha, zona Norte da cidade. Recentemente o molusco foi encontrado em valas da antiga Barragem de Santa Lúcia, zona Sul, no bairro do mesmo nome, antigo criadouro de Biomphalaria glabrata e foco de esquistossomose (Andrade, 1961).

No presente estudo, em continuação a pesquisas sobre a importância epidemiológica dessa espécie em Minas Gerais, foram coletados e examinados exemplares de B. straminea da antiga Barragem de Santa Lúcia, Belo Horizonte, população ainda não estudada, para detectar se os mesmos estavam infectados por Schistosoma mansoni. Parte dos exemplares negativos ou seus descendentes foram usados para criação ou infecção com S. mansoni de duas cepas de Belo Horizonte.

\section{Material e métodos}

No período de 1994 a 1995, foram encontrados e capturados exemplares de Bi omphalaria em valas e poços da antiga Barragem de Santa Lúcia, localizada no bairro do mesmo nome, zona Sul de Belo Horizonte. Nesse local, segundo órgão da Prefeitura Municipal, será implantado o Parque de Santa Lúcia (Figura 1). Os moluscos foram contados, medidos e identificados especificamente pela morfologia interna (Paraense $\&$ Deslandes, 1955).

Foram usados três grupos de cem exemplares, dois coletados no criadouro e outro criado no laboratório. Os exemplares usados mediam 3-7 mm de diâmetro. Cem moluscos coletados nos criadouros e cem criados no laboratório $\left(F_{1}\right)$ foram expostos individualmente a cinqüenta miracídios da linhagem LE de S. mansoni, de Belo Horizonte, mantida no laboratório há mais de vinte anos. Outros cem exemplares foram expostos em massa a cinco mil miracídios da linhagem VGS, obtida de ovos 
encontrados em fezes de escolar de Belo Horizonte. Para a exposição individual, os moluscos foram colocados em placa de plástico branca, com tampa (para cultura de células), contendo divisões cilíndricas com 2,5 ml de água com os miracídios. Para a infecção em massa com a cepa VGS (humana), os moluscos foram colocados em cristalizadores, com tampa, com cerca de $1.500 \mathrm{ml}$ de água, contendo cerca de cinco mil miracídios, cinqüenta por molusco. Para controle, paralelamente foram expostos grupos de cinqüenta exemplares de $\mathrm{B}$. glabrata criados no laboratório, medindo 8 a $10 \mathrm{~mm}$ de diâmetro.

Após a exposição, procedeu-se a transferência dos moluscos para aquários com 38 litros de água desclorada, com sistema de água corrente, aeração e presença de alimento (alface e ração de rato triturada).

Decorridos trinta dias após a infecção, foi feito o primeiro exame, e depois semanalmente até completar setenta dias. Depois desse período, os exemplares negativos foram esmagados entre lâminas de vidro e examinados em microscópio estereoscópico.

A densidade planorbídica nos criadouros foi calculada tomando-se a média de exemplares capturados em dez conchadas (Paulini et al., 1967).

\section{Resultados}

A localização atual dos criadouros de B. straminea em Belo Horizonte é mostrada na Figura 1.

Na Tabela 1 são apresentadas as taxas de infecção experimental dos moluscos coletados em valas e poços da antiga Barragem de Santa Lúcia e de seus descendentes da $\mathrm{F}_{1}$, criados no laboratório. As taxas de infecção foram semeIhantes com as cepas LE e VGS, variando de $10,3 \%$ a $12,0 \%$. As taxas de infecção de B. glabrata, com cinqüenta miracídios da cepa LE por molusco, foram elevadas (Tabela 1). As taxas de mortalidade de $B$. straminea variaram de $12,0 \%$ a $17,0 \%$ com as duas cepas do trematódeo, e a de B. glabrata variou de $0,0 \%$ a $40,0 \%$. Dentre 1.890 moluscos medindo $1-9 \mathrm{~mm}$, coletados no local de 1994 a 1995, nenhum estava naturalmente infectado com S. mansoni ou outros trematódeos.

A densidade planorbídica média, em oito capturas, foi de 16,8 e a variação foi de 2,3 a 92,1 molusco por conchada.

\section{Discussão}

Em Belo Horizonte, o encontro de B. straminea foi registrado anteriormente em valas de sítio próximo ao lago da Pampulha, zona Norte da cidade (Souza et al., 1981a). Os moluscos não estavam naturalmente infectados com S. mansoni, mas as taxas de infecções experimentais com a cepa LE foram de 19,0\% (Souza et al., 1981b), e com as cepas humanas de origem local, MGF eWVS, foram de $11,1 \%$ e $24,6 \%$, respectivamente (Souza et al., 1981b; Souza, 1993).

Durante vários anos não foram encontrados moluscos dessa espécie em outros locais da cidade, apesar de vários levantamentos malacológicos realizados (Souza et al., 1981a).

No presente estudo, os moluscos coletados nas val as da antiga barragem não estavam infectados com S. mansoni, mas ao serem expostos a duas cepas do parasita, procedentes de Belo Horizonte, apresentaram taxas de infecção em torno de $10 \%$ (Tabela 1 ).

A mortalidade de B. straminea após a exposição a miracídios das cepas LE e VGS ficou abaixo de $20,0 \%$. A mortalidade de B. glabrata foi mais elevada $(40,0 \%)$ com a cepa $L E$, devido à maior adaptação parasita-hospedeiro e à carga elevada de miracídos, cinqüenta por molusco, em exposição individual (Souza, 1993). Com a cepa VGS, isolada para esses experimentos, a mortalidade de B. glabrata foi de 0,0\% (Tabela 1).

Como o local do criadouro já foi foco de esquistossomose e tendo em vista a proximidade de uma favela cujas crianças bricam e jogam bola próximo das valas onde são encontrados os moluscos, podendo eventualmente defecar e entrar em contato com a água, existe o risco de contaminação dos moluscos com S. mansoni. De fato, na zona Sul de Belo Horizonte, no

Tabela 1

Infecção experimental de Biomphalaria straminea e Biomphalaria glabrata de Belo Horizonte, Minas Gerais, nos meses de maio e junho de 1995.

\begin{tabular}{lcccc}
\hline $\begin{array}{l}\text { Espécie } \\
\text { de Molusco }\end{array}$ & $\begin{array}{c}\text { Cepa de S. } \\
\text { mansoni }\end{array}$ & $\begin{array}{c}\text { Número } \\
\text { de Moluscos }\end{array}$ & $\begin{array}{c}\text { Porcentagem } \\
\text { de Infecção }\end{array}$ & $\begin{array}{c}\text { Porcentagem } \\
\text { de Mortalidade* }\end{array}$ \\
\hline $\begin{array}{l}\text { B. straminea (F1) } \\
\text { B. glabrata }\end{array}$ & LE & 100 & 12,0 & 17,0 \\
B. straminea & LE & 50 & 66,3 & 40,0 \\
$\begin{array}{l}\text { B. glabrata } \\
\text { B. straminea }\end{array}$ & LE & 100 & 10,3 & 13,0 \\
B.glabrata & VGS & 100 & 100,0 & 40,0 \\
\hline
\end{tabular}

* Trinta dias após a infecção. 
Parque Julien Rien (Figura 1), no bairro Anchieta, ocasionalmente são encontrados moluscos B. glabrata com infecção natural por S. mansoni (Guimarães et al., 1993).

Portanto, Belo Horizonte, onde a transmissão da esquistossomose tem sido feita principalmente por B. glabrata em vários bairros, e em pequena proporção por B. tenagophila no lago da Pampulha (Figura 1), corre o risco de ter mais um foco da parasitose transmitida por $B$. straminea. De fato, se as autoridades municipais, que já foram notificadas sobre a ocorrência do molusco nas valas e planejam construir um parque e lago no local, não tomarem medidas profiláticas adequadas, poderá surgir mais um foco de esquistossomose em zona urbana da região Sul de Belo Horizonte.

\section{Referências}

ALENCAR, J. E.; ROU QUAYROL, M. Z. \& BEZERRA, D. E. A., 1978. A esquistossomose no Ceará, análise do problema atual e sugestões para controle. Revista Brasileira de Malariologia e Doenças Tropicais, 30:99-121.

CARVALHO, O. S.; ROCHA, R. S.; MASSARA, C. L. \& KATZ, N., 1987. Expansão da esquistossomose mansoni em Minas Gerais. Memórias do Instituto Oswaldo Cruz, 82:295-298.

FREITAS, J. R.; JUNQUEIRA, D. U. \& GERKEN, S. E., 1972. Habitats primitivos de hospedeiros do S. mansoni na região de Lagoa Santa (MG). Ciência eCultura, 24:377.

GERKEN, S. E.; ARAÚJO, M. P. T. \& FREITAS, J. R., 1975. Suscetibilidade de Biomphalaria straminea da região de Lagoa Santa (MG) ao Schistosoma mansoni. Revista do Instituto de Medicina Tropical de São Paulo, 17:338-343.

GUIM ARÃES, C. T.; SOUZA, C. P.; CARVALHO, O. S. \& KATZ, N., 1993. Sobre um foco urbano de esquistossomose em área metropolitana da região Sudeste do Brasil. Revista de Saúde Pública, 27:210213.

LUTZ, A., 1934. Transmission du Schistosoma mansoni dans I'Etat de Minas Gerais (Brésil) par le Planorbis centimetralis. Comptes Rendus des Seances de la Societé de Biologie et de Ses Filiales, 116:11491150.

MARTINS, A. V., 1938. Contribuição ao Estudo do Gênero Australorbis Pilsbry. Tese de Cátedra, Belo Horizonte: Faculdade de Medicina.

ANDRADE, R. M., 1961. Estudo ecológico de Austral orbis glabratus no lago artificial de Santa Lúcia, Belo Horizonte, MG, (Brasil). Desaparecimento espontâneo da população de caramujos (Pulmonata, Planorbidae). Revista Brasileira de Malariologia e Doenças Tropicais, 14:29-62.

\section{Agradecimentos}

O presente artigo foi elaborado como parte de um projeto mais amplo sobre o estudo da importância epidemiológica de Biomphalaria straminea em Minas Gerais. O trabalho foi desenvolvido com a colaboração do estudante Paulo Roberto Bastos Ribeiro, bolsista do Programa Institucional de Bolsas de Iniciação Científica - PIBIC - da Fundação Oswaldo Cruz e Conselho Nacional de Desenvolvimento Científico eTecnológico.
PARAENSE, W. L. \& SANTOS, J. M., 1950. Dados negativos sobre a ocorrência do Tropicorbis centime tralis em Belo Horizonte (Estado de Minas Gerais). Memórias do Instituto Oswal do Cruz, 48:199-212.

PARAENSE, W. L. \& DESLANDES, N., 1955. Studies on "Australorbis centimetralis". I. Morphology in comparison with "A. glabratus". Revista Brasileira deBiologia, 15:293-307.

PAULINI, E.; DIAS, E. P. \& FIUZA, H., 1967. Contribuição à epidemiologia da esquistossomose em Belo Horizonte. Revista Brasileira de Malariologia e Doenças Tropicais, 19:571-606.

SOUZA, C. P., 1993. Schistosoma mansoni: Aspectos Quantitativos da Interação Hospedeiro-Parasi to e Desenvolvimento em Biomphalaria glabrata, Biomphalaria tenagophila, e Biomphalaria straminea. Tese de Doutorado, Belo Horizonte: Instituto de Ciências Biológicas, Universidade Federal de Minas Gerais.

SOUZA, C. P.; PEREIRA, J. P. \& RODRIGUES, M. S., 1981a. Atual distribuição geográfica dos moluscos hospedeiros intermediários do Schistosoma mansoni em Belo Horizonte, MG, Brasil. Memórias do Instituto Oswaldo Cruz, 76:383-391.

SOUZA, C. P.; RODRIGUES, M. S. \& ARAÚJO N., 1981b. Suscetibilidade de Biomphalaria straminea (Dunker, 1848), de Belo Horizonte (MG), à infecção por cepas de Schistosoma mansoni. Revista do Instituto de Medicina Tropical de São Paulo, 23:188193.

SOUZA, C. P.; RODRIGUES, M. S.; AZEVEDO, M. L. L. \& ARAÚJO N., 1981c. Suscetibilidade de Biomphalaria straminea (Dunker, 1848), de Minas Gerais, à infecção por Schistosoma mansoni. Revista do Instituto de Medicina Tropical de São Paulo, 23:212216.

SOUZA, C. P.; RODRIGUES, M. S. \& AZEVEDO, M. L. L., 1983. Estudo da potencialidade de Biomphalaria straminea de Minas Gerais, como hospedeiras do Schistosoma mansoni. Memórias do Instituto Oswaldo Cruz, 78:251-256. 